\title{
A Comparative Assessment of Bond Strength of PEEK Crowns to Natural Teeth with Two Different Resin Cements
}

\author{
Nishath Ayesha ${ }^{1}$, Sadiq Mohammed Sabir Ali ${ }^{2}$, Y. Mahadev Shastry ${ }^{2}$, K. Mahendernath Reddy ${ }^{2}$ \\ ${ }^{1}$ Department of Prosthodontics Including Crown and Bridge and Implantology, Kaloji Narayana Rao University of Health Sciences, \\ Telangana, India \\ ${ }^{2}$ Sri Sai Dental College and Hospital, Hyderabad, India
}

Email address:

nishathayesha001@gmail.com (N. Ayesha), sadiqali2000@gmail.com (S. M. S. Ali)

\section{To cite this article:}

Nishath Ayesha, Sadiq Mohammed Sabir Ali, Y. Mahadev Shastry, K. Mahendernath Reddy. A Comparative Assessment of Bond Strength of PEEK Crowns to Natural Teeth with Two Different Resin Cements. International Journal of Dental Medicine. Vol. 6, No. 1, 2020 , pp. 7-12. doi: $10.11648 /$ j.ijdm.20200601.12

Received: May 20, 2020; Accepted: June 20, 2020; Published: August 4, 2020

\begin{abstract}
Statement of problem: For a successful clinical outcome, luting agents should have a high bond strength. Bond failure is still one of the main reasons of restoration failures. Purpose: The purpose of this study was to evaluate tensile bond strength of PEEK with two different resin cements. Materials and methodology: Two self-adhesive cements (RelyX Unicem, G-CEM LinkAce), twenty permanent human premolars were used. In the present experimental study, teeth were prepared to receive copings with a chamfer bur at high speed cooled with an air/water spray. PEEK copings were fabricated and specimens were divided into two groups based on the type of cement used. The tensile load required to de-bond the crowns was measured using universal testing machine with a cross speed of $1 \mathrm{~mm} / \mathrm{min}$. The data was analysed by paired test. Results: The maximum and minimum amounts of bond strength were seen in RelyX Unicem and G-CEM LinkAce cements respectively. Despite this different bond values in cements, paired t test disclosed significant difference between groups in the mean amount of bond strength ( $p$-value=0.05). Conclusion: Regarding the results of the study, it was concluded that G-CEM LinkAce presented higher bond strength.
\end{abstract}

Keywords: G-CEM LinkAce, PEEK, RelyX Unicem, Tensile Bond Strength

\section{Introduction}

The popularity of all-ceramic restorations has increased in recent years due to their superior esthetic appearance, biocompatibility [1] and metal free structure [2]. Some of the materials which have been used till date include alloys, ceramics, zirconia, and high performance polymers. All these, though excellent, have their advantages and disadvantages. Hence, the search has always been on for a better material. One such material, which has made its invasion into dentistry in the recent times, is polyether ether ketone (PEEK). The success of full ceramic restorations in serving for a long time in the mouth depends on the success of bonding between the ceramic, adhesive agent and tooth structure. Although improved mechanical properties are important for the longterm performance of a ceramic material, the clinical success of fixed ceramic prostheses seems to be strongly dependent on the cementation procedure. PEEK is a semi crystalline, high temperature resistant, thermoplastic polymer. The elasticity of this material lies within the range of bone, so it resembles the most natural material. It is biocompatible and chemically stable to nearly all organic and inorganic chemicals [3]. Due to its excellent physical and biological properties, this composite material is used both in general medicine and in dentistry as implant, provisional abutment and implant supported bar or clamp material. PEEK is available in different types such as unfilled, carbon filled and glass filled.

Self-adhesive resin cements are dual cured resin cements which are designed to overcome limitations of both traditional and resin-based cements and simplify the bonding process. Self-adhesive cements simplify bonding procedures and most importantly shortening the "windows of contamination "and better adhesion can be achieved than with a contaminated total-etch cement [4]. Self-adhesive cements can bond to an untreated tooth surface that has not 
been micro abraded or pretreated with an etchant, primer, or bonding agent thus, cementation is accomplished in a single step.

This study has been planned out with the aim of observing the bond strength of resin cements to PEEK.

Thirty freshly extracted premolar teeth were selected for this study. Teeth were prepared according to standard protocol carried on with the diamond cutting burs by producing depth-orientation grooves on the buccal and occlusal surfaces, with a round-end tapered diamond as reference for removing tooth structure. A chamfer finish line of $0.7 \mathrm{~mm}$ depth was prepared using round ended tapered bur.

\subsection{Fabrication of PEEK Copings by CAD-CAM Technique}

Prepared tooth was sprayed with titanium dioxide and scanned. Using a software, a three dimensional virtual image of the specimen was made. The PEEK blank was inserted in the work piece spindle and the door of the milling unit was closed. The milling procedure was completed and PEEK copings were fabricated and polished with $1 \mu \mathrm{m}$ diamond paste, then alumina air blasting was done for 10 seconds with 2.8 bar pressure away from $10 \mathrm{~mm}$ vertical distance using rocatec system to improve the bonding strength of PEEK with resin cements.

\subsection{Resin Bonded Cements}

Specimens were divided into two groups 1 and 2 and bonding was done with two different resin cements. Group 1 were bonded with Rely X Unicem self adhesive universal resin cement. Rely $X$ Unicem is available in an automix syringe form, the working time was 2 minutes and the excess cement was removed in gel state with explorer and then the surfaces were cured for 20 seconds. Group 2 were bonded with G-CEM LinkAce this cement is available in double barrel automix syringe form, through which the cement was directly extruded into the restoration. The working time was 3 minutes 30 seconds by applying normal pressure, on restoration the surfaces were cured for 20 seconds.

\subsection{Universal Testing Machine}

The specimens were placed in the machine between the grips and a device extensometer was attached which was useful to measure tensile tests. The bonded specimens were placed on the table and held with clamp from top and below with upper and lower cross heads of the machine so that the bonded specimens base was parallel to the tensile force direction. Specimens were stressed in an occlusso-gingival direction at a crosshead speed of $1 \mathrm{~mm} / \mathrm{min}$. A jig was attached to the load cell and pulled apart by an upper chain. The maximum load necessary to de-bond was recorded in Newton $(\mathrm{N})$ and calculated in MPa as a ratio of Newton to the cylinder (the calculated bond strength was determined by dividing the strength at which bond failure occurred by the bonding area).

\section{Results}

Statistical Analysis: Data was analyzed by Microsoft excel and graph pad prism software. Data was summarized by Mean \pm SD for continuous data. The comparison between two groups was done by paired t test. All p-values less than 0.05 were considered as statistically significant.

Table 1. Paired t test results

\begin{tabular}{lllll}
\hline Procedures & N & Mean & SD & P value \\
\hline Relyx & 15 & 5.42 & 0.91 & 0.004 \\
g cem & 15 & 6.73 & 1.39 & \\
\hline
\end{tabular}

There was significant difference in the mean tensile strength among two groups $(\mathrm{p}<0.004)$. Paired ' $\mathrm{t}$ ' test showed that group B had significantly higher mean score than group A.

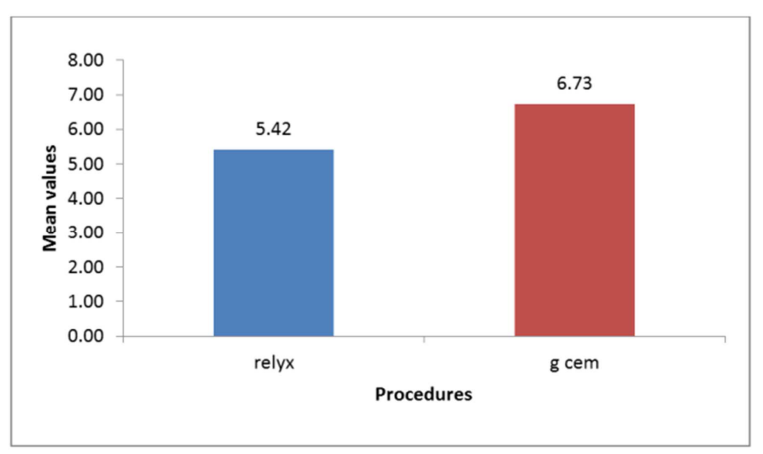

Figure 1. Graphical representation showing mean tensile bong strength values.

Graphical bar diagram representation depicts that G-Cem LinkAce resin cement showed higher tensile bond strength than Rely X Unicem resin cement.

\section{Discussion}

In the present study PEEK copings were fabricated with CAD-CAM technique in which, three dimensional virtual image of the specimen was made. Tamrakar AK et al [5] (2014) stated that the designing of the restoration is done using CAD software, which in turn sends commands to the CAM unit, for fabricating the restoration.

The PEEK surface requires treatment since it has low surface energy. Sandblasting is an efficient method for modifying the surface morphology of materials like metals and polymers. Ourahmoune et al. [6] (2014) studied how sandblasting altered surface morphology in several polymeric materials based on PEEK. The authors noted that sandblastmediated alterations in surface morphology changed wettability and led to an apparent stabilization of roughness parameters after a certain surface treatment time.

They also observed that sandblasting resulted in higher levels of roughness in the fiber-reinforced materials than in the non-reinforced materials.

To achieve optimum retention and bond at the resinsubstructure interface multiple mechanical and chemical 
methods for surface treatment are advocated. The surface of PEEK is very dense and plain, the structure is compact and there are no free chemical connections on the surface which makes it resistant to chemicals. Zhou L [7] (2014) had stated that the incorporation of roughness with 50 microns alumina before surface conditioning is essential to maximize the surface area and increase the bond strength eventually. Therefore 50 microns alumina particles were selected and the PEEK surface was sandblasted as per manufacturer's protocol. Kern [8] (2009) had stated that the incorporation of roughness, is of advantage to achieve adequate bond strength.

To minimize possible surface damage effects as a result of air abrasion at relative high pressures, air-abrasion at lower pressures appears to be an effective method for forming strong durable bonds. It had also been documented by Tetelman [9] (2008) that sandblasting changes the surface morphology of PEEK and enhances the penetration of adhesive cements into composite material and subsequent micromechanical interlocking, resulting in higher bond strength. This is in accordance with the present study where sandblasting aided in increased bond strength.

Previous studies compared the adhesion of resin cements to PEEK with and without surface treatment, Hallmann L et al [10] (2012), and Zhou L et al [7] (2014) tested surface treatments commonly used in dental laboratories, such as sandblasting with alumina and silica coating (Rocatec). Sproesser O et al [1] (2014) tested more experimental surface treatments for dental PEEK such as chemical attack with $98 \%$ sulfuric acid at different exposure times and Stawarczyk B et al [12] (2014) tested cold active gas inert plasma treatment. The association between surface treatments and application of silane agents or adhesives was also tested. These studies concluded that surface treatment of PEEK is needed to obtain adhesion to resin cement. Nevertheless, no established protocol was developed for cementing single crowns or fixed dental prosthesis using a PEEK infrastructure. The manufacturer's recommended cementation protocol is to produce roughness using diamond burs on the inner surface followed by acetone degresing prior to luting system application, however this protocol is difficult to standardize.

Illustrations:

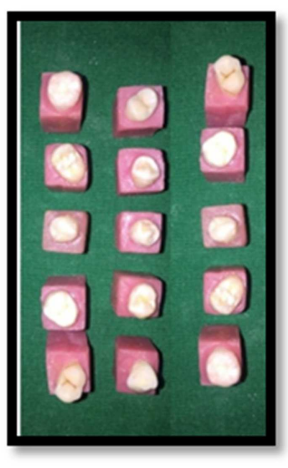

Figure 2. Group 1 specimens.

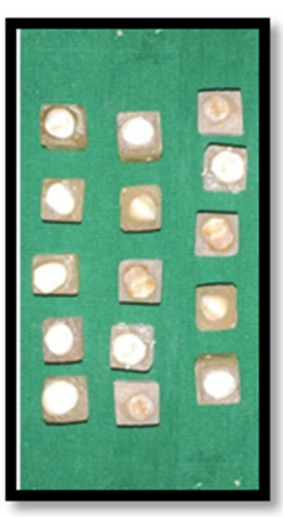

Figure 3. Group 2 specimens.

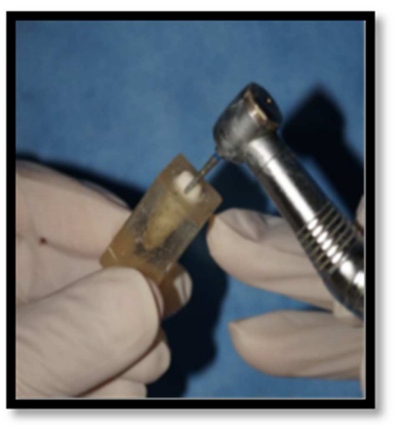

Figure 4. Preparation of natural teeth.

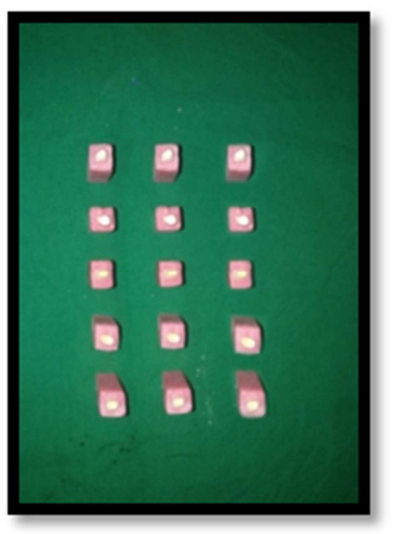

Figure 5. Prepared tooth specimens.

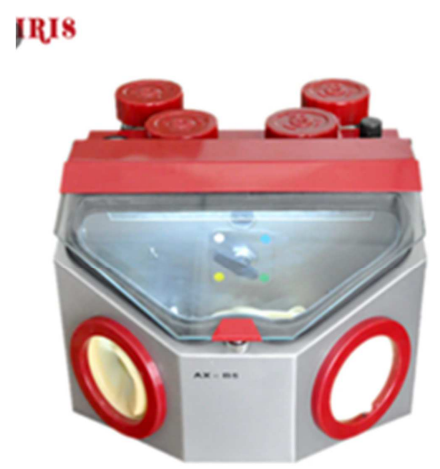

Figure 6. Sandblaster. 


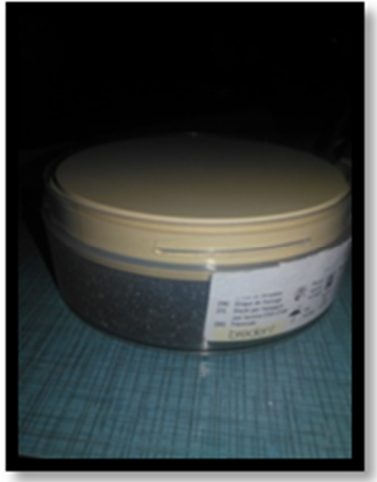

Figure 7. PEEK Blank.

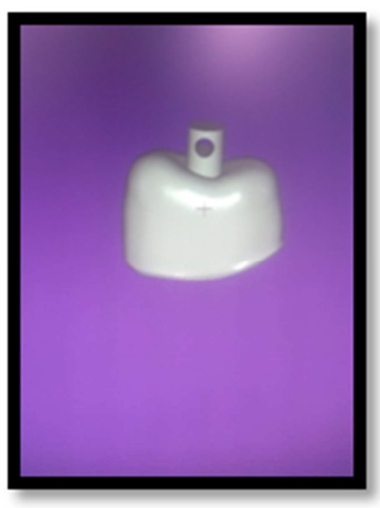

Figure 8. PEEK coping.

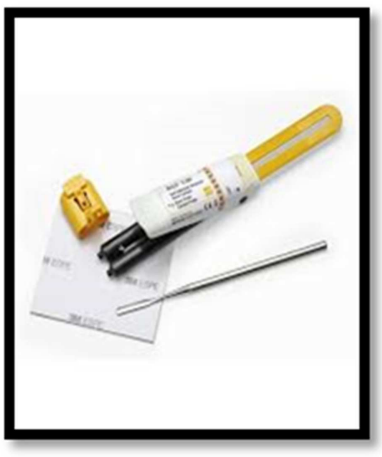

Figure 9. Rely X Unicem.

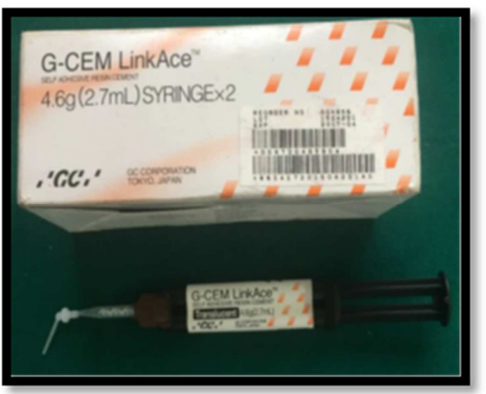

Figure 10. G-CEM LinkAce.

After fabrication of PEEK copings they were sand blasted and divided into two groups, Group 1 and Group 2. Where Group 1 copings were cemented with Rely X Unicem resin cement and Group 2 copings were cemented with G-CEM
Link-Ace resin cement. These are self-curing, transparent, two-paste adhesive resin cement. Equal amounts of resin cements were extruded, mixed for 20 seconds and applied to the intaglio surfaces of PEEK copings. Excess cement was removed using disposable mini brush.

Piwowarczyk A et al [13] (2004) stated that the Selfadhesive dual-cured resin cements are relatively new category of resin luting agents whose organic matrix consists of multifunctional phosphoric-acid methacrylates. These phosphoric-acid groups condition the tooth surface and contribute to adhesion.

The use of Rely X Unicem in operative and prosthodontic applications without etching the enamel or dentin was evaluated by Bishara et al [14] (2005). In a study by De Munck et al [15] (2004) it has been reported that resin based adhesive luting materials were widely used for the fixation of inlays and onlays, crowns, bridges, posts and veneers. The first self-adhesive universal resin cement designed for universal application was Rely X Unicem. It is presented in capsules that can be used for adhesive cementation of indirect ceramic, composite and metal based restoration as well as crowns and bridges (3M ESPE Technical Product Profile 2002).

Sakalauskaite E et al [16] (2008) had stated that the cementation technique is simple and have the potential to decrease postoperative sensitivity. Gerth HU et al [17] (2006) concluded that the resin cements are able to bond to tissues without any previous applications, such as etching, priming, or bonding. Caughman WF et al [18] (2001) stated that the dual-cured resin cements have two polymerizing mechanics, light polymerization and chemical polymerization that leads to extensive use of these cements in practice.

A universal testing machine (UTM) also known as universal tester, materials testing machine or materials test frame was used to test the tensile strength of resin cements in the present study. The bonded specimens were placed in UTM with a cross-head speed of $1 \mathrm{~mm} / \mathrm{min}$. The acrylic resin blocks were held by a collet, allowing the whole system to self-align. Specimens were positioned in the jig with the specimens perpendicular to the loading direction. The jig was attached to the load cell and pulled apart by an upper chain.

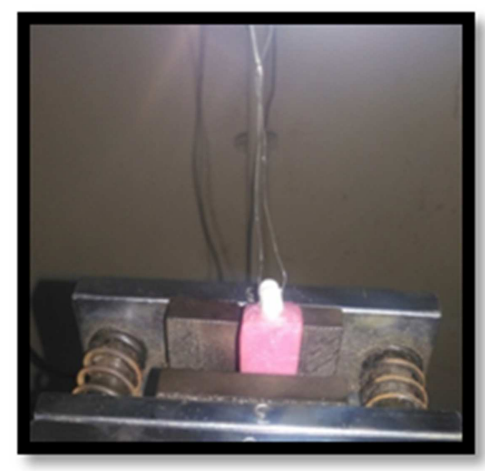

Figure 11. Debonding of specimen. 


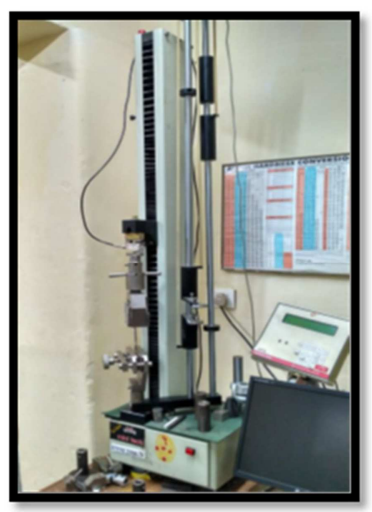

Figure 12. Universal Testing Machine.

The tensile bond strength was calculated.

In the present study the highest tensile bond strength value obtained for RelyX Unicem resin cement was $6.657 \mathrm{MPa}$, whereas the lowest value was $3.946 \mathrm{MPa}$. Highest tensile bond strength value obtained for G-CEM resin cement was $9.56 \mathrm{MPa}$, whereas the lowest value was $4.5 \mathrm{MPa}$, which was in accordance with the study conducted by Piwowarczyk et al [13] (2007) where Rely X showed low bond strength 4.77MPa and G-CEM resin cement showed 6.6MPa this was because of increased chemical interaction of G-CEM with calcium from hydroxyapetite, which may explain the higher bond strength of this cement in this study compared to other resin cements, and the reason for reduced bond strength of Rely X was due to high level of fillers and high viscosity of the cement.

The mean for Rely X Unicem was 0.91MPa with standard deviation of 5.42MPa. The mean for G-Cem was $1.39 \mathrm{MPa}$ with standard deviation of $6.73 \mathrm{MPa}$. In present study the bond strength values could be influenced by numerous parameters related to specimen design or test mechanics which cannot be standardized or might be because of heterogenecity in tooth structure and composition, sample preparation. Our findings indicate that resin cements helps in establishing a durable bond between the tooth and a sandblasted PEEK material. In accordance with study conducted by S. Hattar [19] (2015) Rely X showed $3.70 \pm 6.81 \mathrm{MPa}$ and G-CEM showed $4.48 \pm 5.94 \mathrm{MPa}$, this might be because light-cured cement exhibit a high viscosity and limited penetration. Moreover cements need to be applied with some pressure.

Unicem and G-CEM LinkAce to be 6.0 and $8.8 \mathrm{MPa}$ respectively which is in conformity with our study. This implicates the fact that the value of the bond strength on its own does not always directly correlate to the strength of the material, but the bond strength and the surface area considered together provides a more accurate evaluation.

\section{Conclusion}

Within the limitations of this in vitro study, following conclusions were drawn:

G-CEM LinkAce resin cement had shown highest bond strength than RelyX Unicem resin cement.

\section{References}

[1] Sproesor O, Schmidlin P R, Uhrenbacher J, Roos M, Gernet W, Stawarczyk B. Effect of sulfuric acid etching of polyether ether ketone on shear bond strength to resin cements. $J$ Adhes Dent 2014; 16: 465-72.

[2] Kitakayam S, Toru N, Rena M, Lei Z Masaomi I, Akihiko W, Richard M. Effect of an internal coating technique on tensile bond strength of resin cements to Zirconia ceramics. Dent Materials J. 2009; 28 (4): 446-453.

[3] Kurtz S M, Devine J N, PEEK biomaterials in trauma, orthopedic and spinal implants, Biomaterials 2007: 28; 48454869.

[4] Burgess JO, Ghuman G. A practical guide to the use of luting cements; a prereviewed publication. Originally published as an insert to Dental Economics, 2008.

[5] Tamrakar AK, Manu R, Rizwana M. CAD-CAM in prosthodontics-A futuristic overview Annals of Dental Speciality Vol. 2; 1: 2014.

[6] Ourahmoune R, Salvia M, Mathia TG, Berthel B, Fouvry S, Mesrati N. Effect of sandblasting substrate treatment on single lap shear strength of adhesively bonded PEEK and its composites. In18th international conference on composite materials 2011 (pp. 2-7).

[7] Zhou L, Qian Y, Zhu Y, Liu H, Gan K, Guo J. The effect of different surface treatments on the bond strength of PEEK composite materials. Dent Mater. 2014; 30 (8): e209-15.

[8] M. Kern, A. Barloi, and B. Yang. Surface Conditioning Influences Zirconia Ceramic Bonding JDent Res 2009, 88 (9): 817-822.

[9] Tetelman E D, Babbush C A. A new transitional abudments for immediate aesthetics and function. Implant Dent 2008: 17: 51-58.

[10] Hallmann L, Mehl A, Sereno N, Hämmerle CHF. The improvement of adhesive properties of PEEK through different pretreatments. Appl Surf Sci 2012; 258: 7213-8.

[11] Stawarczyk B, Bähr N, Beuer F, Wimmer T, Eichberger M, Gernet W, Jahn D, Schmidlin PR. Influence of plasma pretreatment on shear bond strength of self adhesive resin cements to polyether ether ketone. Clin oral invest. 2014; 18 (1): $163-70$.

[12] Piwowarczyk A, Lauer HC, Sorensen JA. In vitro shear bond strength of cementing agents to fixed prosthodontic restorative materials. J Prosthet Dent 2004; 92: 265-273.

[13] Bishara SE, Oonsombat C, Soliman M M, Warren JJ, Laffoon $\mathrm{J}$ Ajlouni R. Comparision of bonding time and shear bond strength between a conventional and a new integrated bonding system. Angle Orthod 2005, 75 (2): 237-42.

[14] De Munck. J, Vargas M, Van Landuyt. K, Hikitha. K. 2004. Bonding of an auto adhesive luyting material to enamel and dentin. Dent Mater. 20, 963-971.

[15] Saskalauskaite E, Tam L E, Mc Comb D. Flexural strength, elastic modulus, and $\mathrm{Ph}$ profile of self-etch resin luting cements. J Prosthodont 2008; 17: 262-268. 
[16] Gerth HU, Dammaschke T, ZUCHNER H, Schafer E. Chemical analysis and bonding reaction of Rely X Unicem and Bifix composites-A comparative study. Dent Mater 2006; 22: 934-731.

[17] Caughman WF, Daniel ME, Chan CN, et al. Curing potential of dual -polymerizable resin cements in simulated clinical situations. J Prosthet Dent 2001; 85: 479-484.

[18] Susan Hattar, Muhanad M, Hatamleh, Bond strength of selfadhesive resin Cements to tooth structure. The Saudi Dental Journey 2015. 